\title{
Internal Stress in a Model Elasto-Plastic Fluid
}

\author{
Ooshida Takesh* \\ Department of Applied Mathematics and Physics, Tottori University, JP-680-8552, Japan \\ Ken Sekimotd \\ MSC - CNRS, Université Paris 7, 2 place Jussieu, F-75251 Paris, Franc币
}

(Dated: April 12, 2021)

\begin{abstract}
Plastic materials can carry memory of past mechanical treatment in the form of internal stress. We introduce a natural definition of the vorticity of internal stress in a simple two-dimensional model of elasto-plastic fluids, which generates the internal stress. We demonstrate how the internal stress is induced under external loading, and how the presence of the internal stress modifies the plastic behavior.
\end{abstract}

PACS numbers: 83.60.La, 46.35.+z, 62.20.Fe, 61.20.Lc

The internal stress, also known as the remanent stress in material science, is the stress which is maintained in a material without external mechanical supports. The internal stress is found in both discrete and continuous systems with widely different spatial scales. An examples of macroscale is the "tensegrity" of architecture 1], where the internal balance of the structural elements under tension and those under compression maintains the solidity of the architecture. Its putative counterpart in biological cells has also been proposed 2], where the intracellular cytoskeletal networks are claimed to play the role of the structural elements. In lattice mechanics of solids, we could mention as examples of internal stress the lattice mismatch of crystal growth [3] , the dislocations [4, [5, [6], the phase separation in alloys into the phases of different lattice constants 7]. In polymers, rubbers vulcanized under more than one deformed states (called the permanent set by Flory [8]) would be an example of internal stress. The stresses in those systems, either in atomic or mesoscopic scales, are distributed and establish the mechanical balance among them. The internal stress is thus a very common phenomenon.

As the internal stress is not describable by the monovalent and continuous elastic displacements [4, 5], Eshelby had introduced the "incompatibility tensor" [6] in the context of full three-dimensional lattice defects. Despite the several methods developed so far to describe the internal stress, few studies has been done on the dynamics of the internal stress associated with plastic flows. Also little has been studied on the hysteresis related to the internal stress, especially on the plastic yielding under macroscopic deformations of the system including internal stress [9, 10]. In this Letter we try to shed light on these problems through a simple model of elasto-plastic fluid in which we may use the analogue of the disloca-

\footnotetext{
*Electronic address: ooshida@damp.tottori-u.ac.jp

${ }^{\dagger}$ Electronic address: sekimoto@turner.pct.espci.fr

${ }^{\ddagger}$ Also at: Physico-Chimie Théorique, E.S.P.C.I., 10 rue Vauquelin, F-75231 Paris, France
}

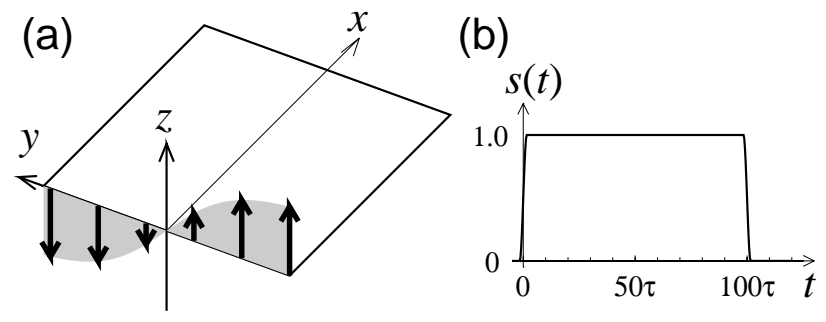

FIG. 1: (a) System's geometry and the initial transient loading on the boundary (thick arrows) at $x=0$. The fluid velocity $\partial u(x, y, t) / \partial t$ lies along the $z$-axis. (b) Time protocol of loading, $s(t)$ (see the text).

tion density [4, [5] to characterize the internal stress. Our model is basically the Bingham model of plastic fluid 11] supplemented with a finite shear compliance. This is a version of generalized Maxwell models of viscoelastic fluid with a longtime relaxation 9, 12. After describing our model, we will show how an internal stress is dynamically brought into a system under the external loading, and how the internal stress is maintained after the removal of the loading. Although the local threshold stress of plastic yielding (called the yield stress) is constant everywhere, the internal stress maintained turns out to be generally subthreshold and inhomogeneous. This implies that the system with yield stress can bear the memory of past operations through the internal stress. Moreover, we will show that the presence of such internal stress modifies the plastic response of the system in a rather intriguing manner.

Our model is constructed as follows. We constrain the fluid to flow in $\| z$ direction while its flow profile may have heterogeneities in the lateral $(x, y)$ directions. We denote the displacement of the fluid along the $z$-axis by $u=u(x, y, t)$. The shear stress components are then essentially represented by the two-dimensional vector, $\boldsymbol{\sigma}=\left(\sigma_{x z}(x, y, t), \sigma_{y z}(x, y, t)\right)$. The evolution of $u$ and $\boldsymbol{\sigma}$ is given by the following isotropic continuum model:

$$
\rho \frac{\partial^{2} u}{\partial t^{2}}=\nabla \cdot \boldsymbol{\sigma}+F^{\mathrm{ext}}
$$




$$
\frac{1}{\mu} \frac{\partial \boldsymbol{\sigma}}{\partial t}+\frac{1}{\eta} \boldsymbol{\Phi}(\boldsymbol{\sigma})=\nabla \frac{\partial u}{\partial t}
$$

where $\nabla \equiv(\partial / \partial x, \partial / \partial y)$ and $F^{\text {ext }}$ is the external force density, which we incorporate only as a boundary loading (see below). $\rho$ and $\mu$ are, respectively, the mass density and the elastic modulus per unit length along the $z$-axis. $\boldsymbol{\Phi}(\boldsymbol{\sigma})$ takes a two-dimensional vector value which is parallel to $\sigma$. This can be regarded as one of the generalized Maxwell models [9, 12], whose original form is recovered by replacing $\boldsymbol{\Phi}(\boldsymbol{\sigma})$ by $\boldsymbol{\sigma}$. The special feature ascribed to the Bingham model 11] is that $\boldsymbol{\Phi}(\boldsymbol{\sigma})$ includes a threshold (yield) stress, $\sigma_{\mathrm{Y}}$, of the plastic yielding:

$$
\boldsymbol{\Phi}(\boldsymbol{\sigma})=\left\{\begin{array}{ll}
0 & \left(\text { for }|\boldsymbol{\sigma}|<\sigma_{\mathrm{Y}}\right) \\
\boldsymbol{\sigma}-\frac{\boldsymbol{\sigma}}{|\boldsymbol{\sigma}|} \sigma_{\mathrm{Y}} & \left(\text { for }|\boldsymbol{\sigma}|>\sigma_{\mathrm{Y}}\right)
\end{array} .\right.
$$

The parameter $\eta$ is the viscosity coefficient per unit length along the $z$-axis. Although not shown, this model can reproduce the one-dimensional propagation of the yielding (i.e. fluidization) front at the speed of the transverse elastic wave, $v_{\mathrm{s}}=\sqrt{\mu / \rho}$. This process of fluidization, which is consistent with a numerical observation [13], is not describable by the classical "Bingham model" where $\mu=\infty$.

Much more spectacular is the behavior in the higher dimensionalities, which can support the internal stress. In our model, we define the vorticity of the internal stress (VIS for short), which we denote by $\omega$, as follows:

$$
\omega \equiv \frac{\partial \sigma_{y z}}{\partial x}-\frac{\partial \sigma_{x z}}{\partial y}
$$

The VIS corresponds to the dislocation density in the continuum description of lattice defects [4, 5], expressed in terms of stress. Our model (2) gives the evolution of the VIS:

$$
\frac{\partial \omega}{\partial t}=-\operatorname{div} \boldsymbol{J}, \quad \boldsymbol{J} \equiv \frac{\mu}{\eta}\left(\begin{array}{c}
\Phi_{y} \\
-\Phi_{x}
\end{array}\right) .
$$

The first equation indicates that $\boldsymbol{J}$ is the conservative flux of $\omega$. Eq. (5) implies that $\omega$ is constant in time where there is no yielding, $\boldsymbol{\Phi}=0$. It also implies that the integral of VIS over a region is invariant if this is surrounded by a non-yielding region.

To study the characteristics of the temporal evolution of the VIS as well as its influence upon the future plastic yielding, we have solved numerically Eqs. (1)-(3). We will focus on the "hydrodynamic" or scaling regime whose spatiotemporal scales are much greater than the viscoelastic timescale $\tau \equiv \eta / \mu$ and the viscoelastic lengthscale $\xi \equiv v_{\mathrm{s}} \tau$. To this end, we take the system of $0 \leq x \leq$ $L_{x}=120 \xi$ and $|y| \leq L_{y} / 2=50 \xi$, and the spatiotemporal mesh sizes, $(\Delta x, \Delta y)=(0.390 \xi, 0.391 \xi)$ and $\Delta t=5 \tau / 16$. We apply a transient inhomogeneous stress $\sigma_{x z}$ on the boundary at $x=0$ with $\sigma_{x z} \gtrless 0$ for $y \gtrless 0$ (Fig. 囵(a)), of (a)
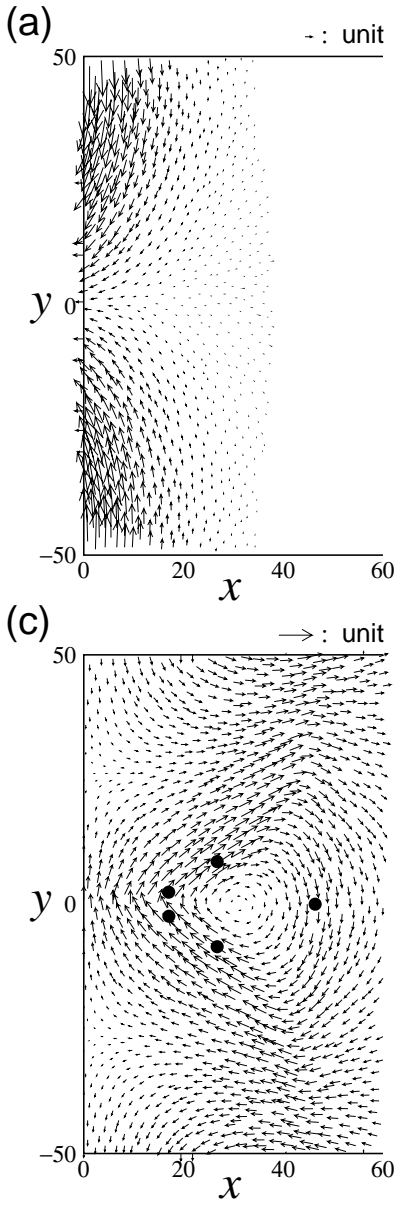

(b)

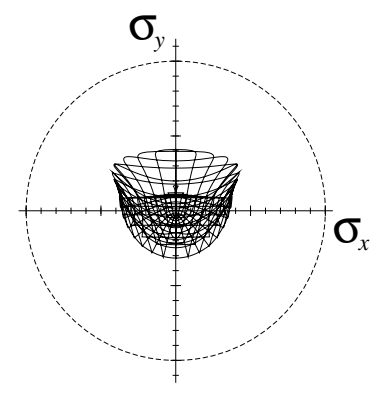

(d)

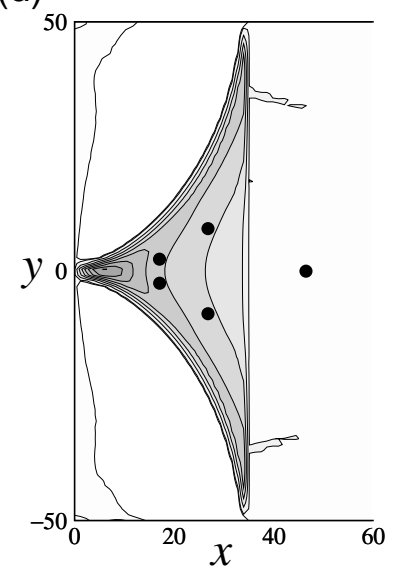

FIG. 2: (a): The flux of the vorticity of the internal stress (VIS), $\boldsymbol{J}$ in units of $\sigma_{\mathrm{Y}} / \tau$, at $t=100 \tau$. (b): The spatially distributed stress components $\left(\sigma_{x z}, \sigma_{y z}\right)$ at $t=500 \tau$ shown in the $\left(\sigma_{x z}, \sigma_{y z}\right)$-plane. The points on the curves give the stress components realized on the rectangular grids in the $(x, y)$-plane, spaced by $15 \Delta x$ and $16 \Delta y$, respectively. The dotted circle represents the yielding threshold, $|\boldsymbol{\sigma}|=\sigma_{\mathrm{Y}}$. (c): The same data as (b) shown on the physical $(x, y)$ plane (in units of $\sigma_{\mathrm{Y}}$ ). (d): The VIS, $\omega$, for the same data as (b), shown by gray scales and contours. (Only the range of $0 \leq x \leq 60 \xi$ is shown in $(\mathrm{a}, \mathrm{c}, \mathrm{d})$, with the axes labeled in units of $\xi$.) The thick dots $(\bullet)$ in $(\mathrm{c}, \mathrm{d})$ at $x=17.0 \xi, 26.8 \xi(y>0), 26.8 \xi(y<0)$, and $46.4 \xi$ indicate the locations where the subsequent yield starts under the specific uniform external shear stresses: $\left(\sigma_{x z}^{\text {ext }}, \sigma_{y z}^{\text {ext }}\right)=$ $\sigma^{\text {ext }}(\cos \theta, \sin \theta)$ with $\theta=\pi / 2,0, \pi,-\pi / 2$, respectively (see the text).

the profile, $\left.\sigma_{x z}\right|_{x=0}=\sigma_{\max } s(t) \tanh \left(y / L_{0}\right)$. Here $\sigma_{\max }=$ $10 \sigma_{\mathrm{Y}}, L_{0}=50 \xi$, and $s(t)$ is a slightly smoothened unit step-function, taking the value of unity only during the initial period of about $100 \tau$, with a smoothing period of approximately $1.5 \tau$ (Fig. 1(b)). The conditions on the other boundaries have been adjusted to mimic approximately reflection-free walls 14]. For the temporal evolution we have used the two-step Lax-Wendroff (LW) scheme. The validity of this second-order scheme for the 
present problem has been checked in the one-dimensional situation by comparing it with the Cubic-Interpolated Propagation (CIP) scheme of the third-order precision.

Fig. 2(a) shows the snapshot of the flux of the VIS, $\boldsymbol{J}$, at $t=100 \tau$, the time at which the external loading is removed. The direction of $\boldsymbol{J}$ is governed by that of $\boldsymbol{\sigma}$ : from (3) and (5), we have $\boldsymbol{J} \perp \boldsymbol{\sigma}$ with $|\boldsymbol{\sigma}|>\sigma_{\mathrm{Y}}$.

Figs. 2(b) and 2(c) show the distribution of the internal (or remanent) stress, $\boldsymbol{\sigma}=\boldsymbol{\sigma}^{\mathrm{int}}(x, y) \equiv\left(\sigma_{x z}^{\mathrm{int}}(x, y)\right.$, $\left.\sigma_{y z}^{\text {int }}(x, y)\right)$, on the $\left(\sigma_{x z}, \sigma_{y z}\right)$-plane and on the $(x, y)$ plane, respectively, at $t=500 \tau$, when all the plastic flow has died out and also the elastic waves have already left the system through the reflection-free boundaries (therefore $\nabla \cdot \boldsymbol{\sigma}^{\text {int }}=0$ ). Evidently, the amplitude of the internal stress is by no means stuck to the threshold value, $\sigma_{\mathrm{Y}}$, but is distributed around zero. (The maximum value of $|\boldsymbol{\sigma}|$ in Fig. 2(b,c) is about $0.515 \sigma_{\mathrm{Y}}$. ) This is because the stress field is elastically redistributed after the plastic deformations so that it finally satisfies the static balance, $\nabla \cdot \boldsymbol{\sigma}^{\text {int }}=0$. In fact it is easy to show, from this condition, that the spatial average of $\boldsymbol{\sigma}$ should be strictly equal to zero in the final state with no forces on the boundaries.

Fig. 2(d) shows the distribution of the VIS, $\omega$ (see (4) calculated from the data of Fig.2(b,c). As compared with the spatial distribution of the internal stress, the VIS reveals a peculiar localized peak, which is dislocated from the center of the noticeable large-scale circular distribution of the stress itself [15]. In the present setup of loading, the VIS $(\omega)$ is initially distributed rather broadly in the $y$-direction (not shown), reflecting the smooth spatial profile of the external loading at the boundary of $x=0$. However, the flux $\boldsymbol{J}$, which drives the VIS symmetrically with respect to the $x$-axis (see Fig. 2(a)), acts to localize the VIS near this axis. The fan-shaped spatial characteristics of $\omega$ in Fig. 2(d) suggests an approximate selfsimilarity in the distribution of the VIS. This self-similar nature can also be seen in the spatial Fourier spectrum of $\omega$ (Fig. 3), which shows a power-law regime in the intermediate range of wave numbers.

The state shown in Fig. 2(b,c,d) retains the memory of the plastic flow in the past in the form of the VIS. This memory in turn influences the plastic response to the future external loading. As an example, if we apply quasi-statically a uniform external stress $\boldsymbol{\sigma}=\sigma^{\text {ext }} \hat{\boldsymbol{t}}(\theta)$, where $\sigma^{\text {ext }}>0$ and $\hat{\boldsymbol{t}}(\theta)=(\cos \theta, \sin \theta)$, to the above mentioned state with an internal stress, the yield then occurs with a threshold amplitude of $\boldsymbol{\sigma}^{\text {ext }}$ which is generally smaller than $\sigma_{\mathrm{Y}}$. Moreover, both the location $(x, y)$ and the threshold amplitude of $\boldsymbol{\sigma}^{\text {ext }}$ at which the yield starts depends on the orientation $\theta$ of the applied uniform stress. As a demonstration, the thick dots in Fig. 2 $(\mathrm{c}, \mathrm{d})$ indicate such locations for the external shear stress of several orientations. It is interesting to note that the locations where the yield starts is well distant from the region where VIS is concentrated. Moreover, the location of the first yield as a function of $\theta$ is not necessarily continuous nor single-valued, as seen by the two thick dots at $x=17.0 \xi$ for the external stress: $\left(0, \sigma_{y z}^{\text {ext }}\right)$ with

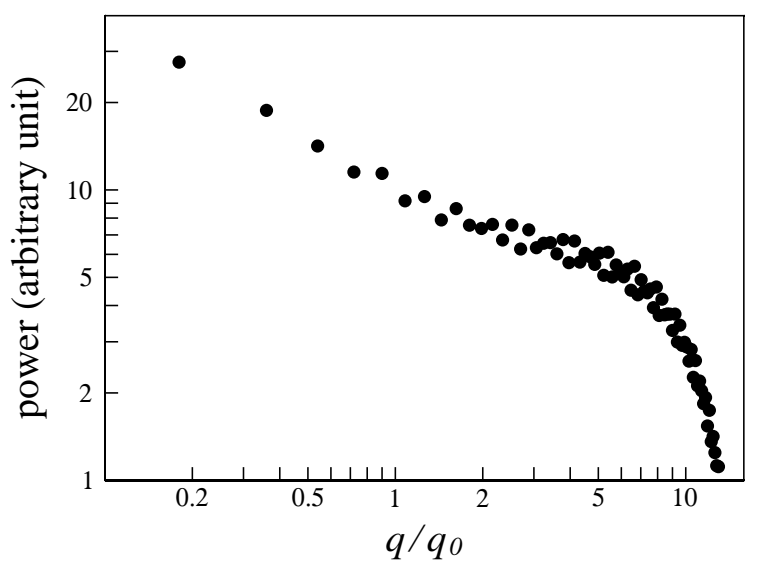

FIG. 3: The log-log plot of the two-dimensional spatial Fourier spectral intensity of $\omega$, integrated over the angle, vs the amplitude of the wave numbers (in units of $q_{0} \equiv(2 \xi)^{-1}$, the wave number beyond which the yielded fluid has no propagative modes). The exponent of the hydrodynamic regime $\left(q<q_{0}\right)$ is read out to be about $-1 / 3$.

$\sigma_{y z}^{\mathrm{ext}}>0$.

We will describe how the system with the internal stress, $\boldsymbol{\sigma}^{\text {int }}(x, y)$ (see Fig. 2(b,c)), starts the plastic yield under further quasi-static application of a uniform external stress, $\boldsymbol{\sigma}=\sigma^{\mathrm{ext}} \hat{\boldsymbol{t}}$. First we note that, before the yielding, the uniform external stress is simply superposed onto the internal stress, $\boldsymbol{\sigma}^{\text {int }}(x, y)$, to give the actual stress as $\boldsymbol{\sigma}^{\text {int }}(x, y)+\sigma^{\text {ext }} \hat{\boldsymbol{t}}(\theta)$. The external stress at the plastic yielding, which we denote by $\sigma_{\mathrm{Y}}^{\text {ext }}$, is, therefore, the solution of the following equation:

$$
\max _{(x, y)}\left|\boldsymbol{\sigma}^{\mathrm{int}}(x, y)+\sigma_{\mathrm{Y}}^{\mathrm{ext}} \hat{\boldsymbol{t}}(\theta)\right|=\sigma_{\mathrm{Y}},
$$

where $\theta$ is fixed. Here those coordinates $(x, y)$ that realize the maximal value on the left hand side tell the location (or locations) at which the plastic yielding starts. The threshold external stress thus obtained plays the role of apparent yield stress of the system with internal stress. Eq. (66) is easily interpreted and solved graphically: As shown in Fig. 2(b), the internal stress $\boldsymbol{\sigma}^{\text {int }}(x, y)$ on the $\left(\sigma_{x z}, \sigma_{y z}\right)$-plane forms a closed domain. The superposition of an external uniform stress, $\sigma^{\text {ext }} \hat{\boldsymbol{t}}(\theta)$, corresponds to a parallel displacement of this domain by $\sigma^{\text {ext }} \hat{\boldsymbol{t}}(\theta)$. If we increase $\sigma^{\text {ext }}$, the displaced domain should eventually touch from inside the circle of the yielding condition, $|\boldsymbol{\sigma}|=\sigma_{\mathrm{Y}}$ (the dotted circle in Fig. 2(b)).

The above graphical solution immediately leads us to the following three qualitative conclusions: (i) The amplitude of the apparent yield stress $\sigma_{\mathrm{Y}}^{\text {ext }}$ is generally less than $\sigma_{\mathrm{Y}}$, the value expected in the internal-stress-free system. (ii) If the apparent yield shear stress in one direction is less than $\sigma_{\mathrm{Y}}$, then it is true also in the opposite direction, to meet with the requirement of vanishing spatial average of the internal stress as mentioned above. (iii) The yielding point $(x, y)$ which solves (6) can be discontinuous as the function of the direction of external 
shear, $\theta$. In fact, if we parallelly displace the domain of $\boldsymbol{\sigma}^{\text {int }}(x, y)$ by varying $\theta$ while adjusting the value of $\sigma^{\text {ext }}$ so that the domain remains tangent to the circle of $|\boldsymbol{\sigma}|=\sigma_{\mathrm{Y}}$, the tangential point can undergo jumps. We expect a similar type of discontinuity to appear for the other criteria of plasticity as long as they share a similar mathematical structure to (6).

In this Letter we have defined and analyzed the internal stress in a continuous model of elasto-plasticity, with the emphasis on the dynamics of the vorticity of the internal stress $(V I S)$ as the source of the internal stress. Some of the insights obtained from our analysis could be generalized to other rheological models, and also to the models of higher dimensionality: (1) the internal stress after the yielding can be inhomogeneous, (2) the amplitude of the internal stress is generally less than the threshold value $\sigma_{\mathrm{Y}}$, being redistributed so that the internal balance of elastic force is established, (3) the apparent threshold yield stress under subsequent global shear deformation is different from $\sigma_{\mathrm{Y}}$ and anisotropic. In order to verify and develop these ideas, it is, firstly, essential to recognize the quantity like VIS which is responsible to the internal stress and is invariant under elastic deformations. Secondly, it is important to analyze how such quantity is created and kept as the memory reflecting the past history of the system's plastic flow. Finally, we should analyze the consequences of the inhomogeneous internal stress such as the apparent threshold yield stress under the subsequent global loading.

To be more realistic, the simple constitutive equation of the "Bingham type" should be modified so that it represents the plastic yield as a slow dynamics involving the effect of temperature and the timescales of the observation and the operation [9, 13, 16]. Since the definition of VIS is independent of the inertia effect, the concept of VIS should be applicable to other rheological models in which the momentum can be ignored while some internal degrees of freedom of the material are relevant. Of particular interest would be the relation between internal stress and slow relaxation: the (extended) distribution of relaxation time observed through the aging or creep experiments 17] could partly be attributed to the distribution of the internal stress. In systems consisting of discrete elements, like granular materials [10], a macroscopically homogeneous shear will be sufficient to cause internal stress among those elements, due to local anisotropy of elasto-plastic interactions. More quantitative analysis on this point could be made in future. As mentioned in the introductory part, the internal stress is found in many different systems not being limited to the rheological fluids. Systems composed of active elements (ex. biological cells), where the internal stress may be generated by the medium itself, would be highly of interest in this aspect.

T. O. thanks Shin-ichi Sasa and Hiroshige Matsuoka for fruitful discussions. K. S. has been partially supported by the Yamada Science Foundation. The authors also acknowledge A. Ajdari for having drawn our attention to [12] during revision of our manuscript.
[1] R. B. Fuller, Tensile-integrity structures. United States Patent 3,063,521 (1962), Filed 31 Aug. 1959, Granted 13 Nov. 1962.

[2] Donald E. Ingber, Scientific American 278, No.1 (1998) 48-57.

[3] J.C. Bean, Science 230 (1985) 127; C.A.C. Ball J.H. van der Merve, in Dislocation in Solids, Ed. F.R.N. Nabarro, North Holland Publ. Co., Amsterdam 1983; E. Granato, J. M. Kosterlitz, S. C. Ying Phys. Rev. B 393185 (1989).

[4] See, for example, A. M. Kosevich, E. M. Lifshitz, L. D. Landau and L. P. Pitaevskii, Theory of Elasticity. (Butterworth-Heinemann, 1986) §29.

[5] J. Friedel, Dislocations. Addison-Wesley Pub. Co., Inc., Reading, Massachusetts 1963.

[6] J. D. Eshelby, The continuum theory of lattice defects, Solid State Physics, Vol.3, pp. 79-144 (Academic Press Inc., New York, 1932).

[7] J. W. Cahn, Acta Metallurgica 14, 83 (1966).

[8] P. J. Flory, Trans. Faraday Soc. 56, 722 (1960).

[9] Y. Miyamoto, K. Fukao, H. Yamao and K. Sekimoto, Phys. Rev. Lett. 88, 225504 (2002).

[10] A. Nakahara and Y. Matsuo, J. Phys. Soc. Jpn. 74, 1362
(2005); cond-mat/0501447 v2.

[11] E. C. Bingham, Fluidity and Plasticity (McGraw-Hill, New York (1922)).

[12] C. Derec, A. Ajdari, F. Lequeux. Eur. Phys. J. E 4, 355361 (2001); G. Picard, A. Ajdari, F. Lequeux, L. Bocquet. Phys. Rev. E 71, 010501(R) (2005).

[13] F. Varnik, L. Bocquet, J.-L. Barrat, J. Chem. Phys. 120, 2788 (2004): cond-mat/0309089; F. Varnik, L. Bocquet, J.-L. Barrat, L. Berthier, Phys. Rev. Lett. 90, 095702 (2003).

[14] T. Ooshida and K. Sekimoto, to be submitted.

[15] J.P. Wittmer, A. Tanguy, J.-L. Barrat, and L. J. Lewis, Europhys. Lett. 57, 423 (2002); A. Tanguy, J.P. Wittmer, F. Leonforte, and J.-L. Barrat, Phys. Rev. B 66, 174205 (2002); F. Leonforte, A. Tanguy, J.P. Wittmer, and J.-L. Barrat Phys. Rev. B 70, 014203.

[16] A. Aradian and M. Cates, cond-mat/0310660. (AIP Conference Proceedings vol. 708, pp. 84-87. M. Tokuyama and I. Oppenheim, eds. Melville, NY, 2004.)

[17] D. A. Weitz. Nature (News and views) 410, 32-33 (2001). 